


\section{Noninvasive ventilation as an adjunct to exercise training in patients with chronic respiratory disease Educational aims}

1 To describe the potential benefits of noninvasive positive-pressure ventilation (NIV) as an adjunct to exercise training in selected patients with chronic obstructive pulmonary disease (COPD) or chest wall restriction.

I To outline the mechanisms by which NIV may improve exercise performance in selected patients with COPD or chest wall restriction.

1 To identify characteristics of patients with chronic respiratory disease who are more likely to respond to NIV-assisted training.

I To highlight major practical considerations for implementing NIV-assisted training in an individual with COPD or chest wall restriction.

\section{Summary}

Severe exertional dyspnoea is a major impediment to physical activity and adversely affects quality of life for many individuals with chronic respiratory disease. Although exercise can improve cardiorespiratory and peripheral muscle responses, training at sufficient intensity and duration may be difficult for the very breathless, deconditioned patient. Consequently, strategies to optimise training for such individuals are gaining interest. NIV is one such option and there is a growing body of evidence outlining its benefits in selected patients. However, the technique is not without its constraints and problems. An understanding of the mechanisms underlying improvements in exercise performance with NIVassisted training is important in targeting appropriate candidates for intervention and identifying the best therapy to achieve the maximum benefit.

Severe exertional dyspnoea is a major disabling symptom for individuals with chronic respiratory disease secondary to COPD or chest wall restriction, limiting physical performance and adversely affecting quality of life. Although the mechanisms responsible for dyspnoea and reduced exercise tolerance will vary depending on the underlying primary disorder, alterations in pulmonary mechanics along with reductions in respiratory muscle strength and endurance contribute to respiratory muscle fatigue and limit ventilatory capacity. With reduced physical activity, further peripheral muscle deconditioning occurs, promoting exertional dyspnoea and fatigue and causing less activity.

The intention of exercise training is to improve exercise capacity by decreasing the ventilatory requirement for a given work-rate and to reduce lactic acidosis. However, training needs to be performed at an adequate intensity and for a sufficient duration if such benefits are to be obtained. For individuals
A.J. Piper1,2,3

C. Menadue ${ }^{1,3}$

${ }^{1}$ Respiratory Failure Service, Dept of Respiratory and Sleep Medicine, Royal Prince Alfred Hospital, Camperdown, ${ }^{2}$ Woolcock Institute of Medical Research, Glebe, and ${ }^{3}$ Discipline of Physiotherapy, University of Sydney, Lidcombe, Australia.

\section{Correspondence}

A.J. Piper

Respiratory Failure Service Dept of Respiratory and Sleep Medicine

Level 11, EBlock

Royal Prince Alfred Hospital

Missenden Road

Camperdown 2050

Australia

E-mail:ajp@med.usyd.edu.au

Fax: 61295157196

\section{Provenance}

Commissioned article, peer reviewed

\section{Competing interests}

In the past 5 years, A.J. Piper has received honorara for lecturing and organising educational sessions from ResMed Asia Pacific (Bella Vista, Australia), Weinmann (Hamburg, Germany) and Respironics Australia (Sydney, Australia), and received travel support from Weinmann. A.J. Piper has also previously received equipment support from Respironics and Air Liquide (Sydney, Australia). 
with chronic respiratory disease characterised by severe breathlessness, achieving a sufficiently high-intensity training stimulus can be challenging. Consequently, there is increasing interest in strategies such as NIV that could be used as an adjunct to exercise training to reduce ventilatory load during exercise and improve exercise capacity in patients with respiratory insufficiency. Recent evidence-based guidelines concluded that NIV may confer an immediate after-rehabilitation benefit in improving exercise tolerance in selected patients with more advanced COPD [1]. However, currently available data show a wide individual variability in response to exercise with NIV [2-4], stressing that this technique is not suitable as a routine intervention and should be targeted at those individuals who are "responders". The purpose of this article is to review current evidence for the effectiveness of this technique in enhancing exercise performance and to highlight important practical considerations and limitations when using NIV.

\section{Nocturnal ventilatory support and exercise performance}

While nocturnal ventilatory support was initially introduced with the intention of reversing respiratory failure and reducing symptoms related to hypoventilation, a number of studies have reported accompanying improvements in daytime exercise tolerance and physical activity in the absence of formal exercise training [5-7]. In a study of patients with chronic hypercapnic respiratory failure (CRF) with mixed aetiology, a relationship between the severity of daytime hypercapnia and daily activity was found [6]. Furthermore, significant improvements in activity levels occurred following the introduction of NIV, correlating with a fall in arterial carbon dioxide tension $\left(\mathrm{Pa}_{1} \mathrm{CO}_{2}\right)$ [6]. Similarly, in a group of patients with CRF due to restrictive thoracic disease, 3 months of nocturnal NIV produced a marked increase in endurance time during exercise tests involving respiratory and peripheral muscles. In contrast, in a matched group of controls assigned to normal care, no significant improvement in any test was observed [7]. Reports of the beneficial effects of nocturnal ventilatory support on exercise endurance have predominantly come from studies involving patients with restrictive chest wall disorders; although in a randomised crossover trial, significant improvements in walking distance were seen in patients with severe cystic fibrosis when using nocturnal bilevel support compared with the control (air) arm [5]. In contrast, a number of randomised trials investigating the addition of nocturnal NIV to long-term oxygen therapy (LTOT) in severe hypercapnic COPD found no difference in exercise capacity in the NIV and LTOT group compared with LTOT alone $[8,9]$. In a more recent study examining endurance time during constantload exercise, non-COPD patients with hypercapnia increased their exercise time signifcantly following 3 months of NIV, in contrast to those with COPD where no significant change was observed [10]. These variations in findings suggest that the impact of nocturnal NIV alone on exercise capacity may differ depending on the primary underlying disorder [10]. In addition, even when exercise performance is improved, the level of daily activity in patients with chronic respiratory disease using nocturnal NIV remains well below that seen in age-and sex-matched healthy subjects [6]. This, along with the lack of improvement in exercise tolerance in COPD patients using nocturnal NIV, highlights the important role of exercise training in patients with severely reduced ventilatory capacity.

In a randomised trial, GARROD et al. [11] looked at the use of NIV in patients with COPD from a different viewpoint, proposing that nocturnal NIV provided in conjunction with an exercise training programme could lead to greater improvements in exercise capacity and health status than training alone. Importantly, these patients were recruited on the basis of their severe COPD and limited exercise tolerance due to dyspnoea, and not nocturnal sleep disturbance or daytime hypercapnia. If patients were unable to sleep with the ventilator they were advised to use therapy for $\geq 6 \mathrm{~h}$ during the day. Individuals assigned to NIV in addition to exercise training demonstrated improvements in exercise tolerance and quality of life over and above changes seen in the group undergoing exercise training alone. This was despite poor compliance with NIV, with only $29 \%$ using therapy for $>4 \mathrm{~h}$ per day. More recently, DuIverman et al. [12] randomised 72 patients with chronic hypercapnic respiratory failure and severe COPD to NIV in addition to pulmonary rehabilitation or pulmonary rehabilitation alone to determine any additional impact on health-related quality of life, functional status and gas exchange. Compliance with nocturnal NIV therapy improved from the earlier study of GARROD et al. [11], with a median use of $7.7 \mathrm{~h}$ per day. Although NIV provided no additional improvement in the total score of the Chronic Respiratory Questionnaire (CRQ; the 
primary end-point of the study), there were improvements in other outcome measures including the CRQ fatigue domain, the Maugeri Respiratory Failure questionnaire (MRF-28), daytime $\mathrm{Pa}_{1} \mathrm{CO}_{2}$ and daily activity levels. Although neither study was designed to investigate the mechanisms by which NIV used during sleep could improve the response to daytime exercise training, it was proposed that the benefits seen may have arisen from improved respiratory muscle function through unloading of these muscles or from improved gas exchange [11, 12]. These findings along with recent comparison data [10] emphasise the need to incorporate exercise training into the management of patients with COPD using nocturnal NIV in order to achieve additional exercise benefits.

\section{Nonivasive ventilation during exercise Lower-limb exercise \\ Acute responses to NIV: COPD}

The immediate effects of NIV during treadmill running and cycling have been addressed in a number of studies in patients with COPD $[2,3,13-16]$. In a meta-analysis, VAN'T HuL et al. [17] found that NIV delivered in the form of continuous positive airway pressure (CPAP), inspiratory pressure support (IPS) or proportional assist ventilation (PAV), led to acute improvements in exertional dyspnoea and exercise endurance. However, the degree of improvement varied considerably between individuals, with some patients only marginally improving while others more than doubled their exercise endurance $[2,3]$. At the other extreme, in a randomised study Hıснсоск et al. [18] found no benefit from using NIV during treadmill walking, with a marked reduction in the distance walked during sessions with NIV even though tidal volume and minute ventilation increased significantly compared with walking with a mouthpiece alone. Similarly, JoHnson et al. [13] found that in a group of COPD patients unfamiliar with NIV, exercise performance did not immediately improve when compared with unassisted exercise, with the NIV group exercising for an average of $1 \mathrm{~min}$ less when using the machine.

In addition to improvements in dyspnoea and exercise endurance, other favourable physiological changes have been identified during the acute application of NIV during exercise. These include an improved breathing pattern as reflected by a reduction in respiratory rate and increase in tidal volume [14], increased minute ventilation at the

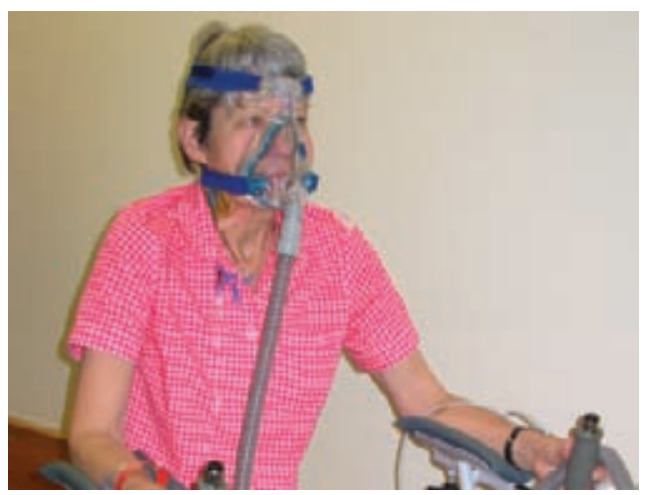

end of exercise [16] and reduced exercise-induced lactic acidosis [19]. These changes are consistent with the substantial reduction in respiratory muscle loading that has been observed with the application of NIV during exercise $[3,15,16]$.

Acute responses to NIV: chest wall restriction Individuals with chest wall restriction commonly have limited ventilatory reserve and severe exertional dyspnoea [4]. Although low-flow supplemental oxygen during walking has been shown to reduce dyspnoea, decrease oxygen desaturation and shorten recovery time compared with sham oxygen (air), no improvement in exercise performance was demonstrated in patients with stable kyphoscoliosis [20].

In patients with scoliosis, maximum exercise ventilation is reduced proportional to the loss in vital capacity while work of breathing is increased [21]. Since NIV can increase minute ventilation while reducing inspiratory effort, its use during exercise training could theoretically improve endurance and the intensity of training. To test this hypothesis, HIGHсock et al. [21] randomised eight patients with severe scoliosis to three walking conditions: unencumbered; with mouthpiece alone; and during NIV with mouthpiece. All testing was performed without supplemental oxygen. While ventilation was increased during exercise with NIV, exercise capacity did not improve, and the authors concluded that there was no role for NIV as an adjunct to exercise training in patients with scoliosis. In contrast, other studies have reported benefit $[4,22,23]$. TsuBol et al. [22] studied seven patients with chest wall restriction secondary to tuberculosis sequelae during constant work rate. Exercise tests were performed on a cycle ergometer in the supine position over a 2-day period: spontaneous breathing on air and NIV on day 1 ; and spontaneous breathing on oxygen and NIV sequentially on day 2 . The order of days was randomised. Noninvasive volume preset ventilation during exercise improved blood gases, reduced breathlessness and improved exercise endurance. 
No improvement in endurance was seen with oxygen therapy alone, while the combination of NIV with supplemental oxygen led to the greatest improvements in exercise endurance. In a recent randomised crossover study, Borel et al. [4] demonstrated that the acute use of NIV during cycling permitted the extension of constant-workload exercise time by $71 \%$. A significant correlation between the increase in exercise endurance and the reduction in breathlessness during NIV was noted $[4,22]$. Using a similar randomised cross over design in eight patients with kyphoscoliosis, NIV was shown to alleviate hypoventilation associated with light cycling, while reducing dyspnoea and perceived effort [23]. However, as seen in patients with COPD the immediate response to NIV-assisted exercise was variable, with some patients greatly improving exercise duration while others were slightly impaired [4].

\section{NIV as part of a training programme: COPD}

To date seven randomised trials evaluating the effect of NIV administered during an exercise training programme in people with COPD have been published (table 1). The majority of these trials demonstrated that training with NIV allowed patients to achieve significantly higher training intensities [24-26], and greater improvements in exercise performance compared with unassisted training $[13,24-28]$. Only one study has reported no significant benefits [29]; however, patients had slightly less severe airflow obstruction than

Table 1. Summary of randomised trials evaluating NIV as an adjunct to exercise training

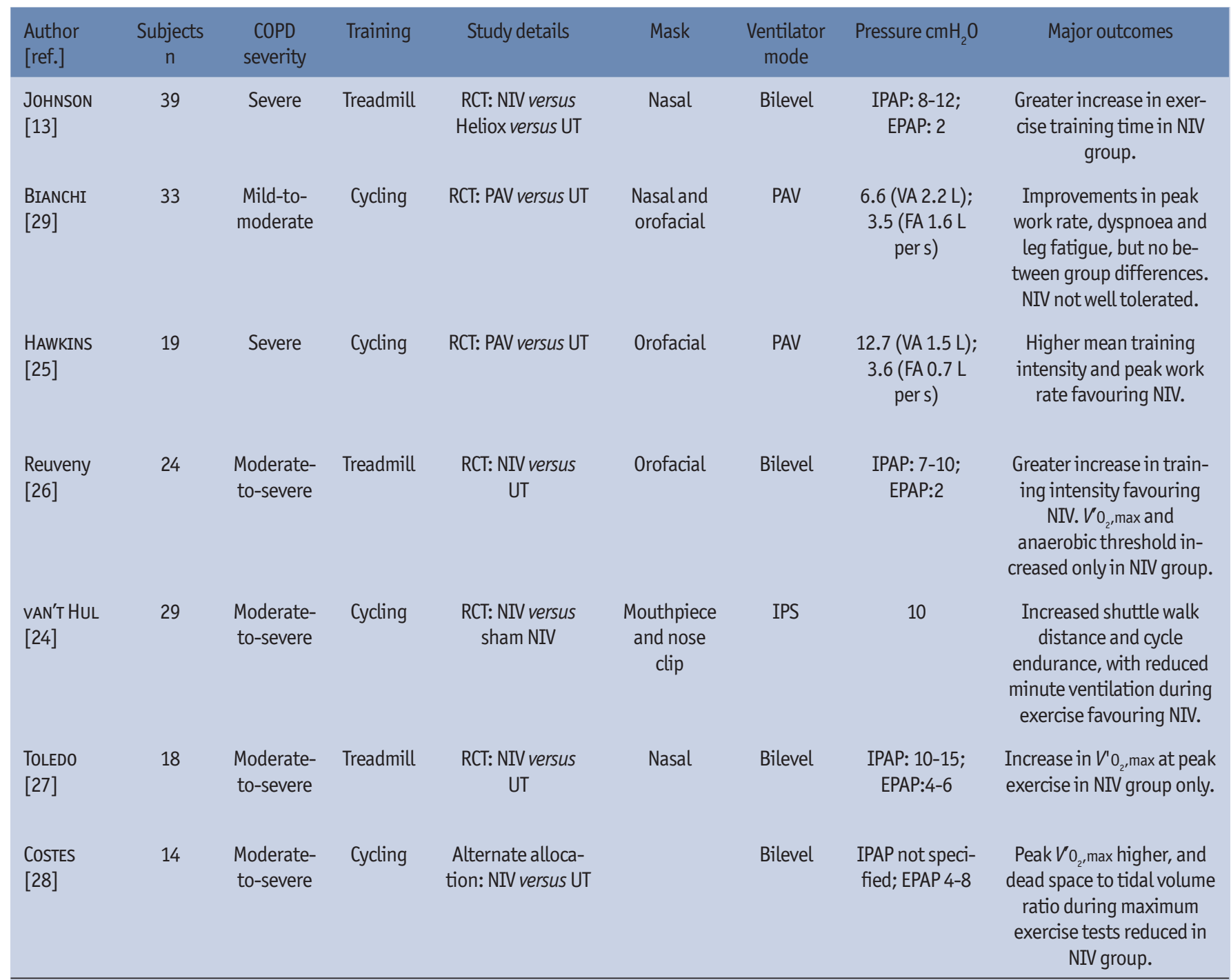

RCT: randomised controlled trial; UT: usual training; IPAP: inspiratory positive airway pressure; EPAP: expiratory positive airway pressure; VA: volume assist; FA: flow assist; $V{ }^{\prime}{ }_{2}$ max: maximal oxygen uptake. 
studies reporting benefits. Interestingly, this same group of investigators previously found that NIV acutely reduced dyspnoea and improved endurance time [14], although the patients included in the earlier trial were hypercapnic with moderateto-severe airflow limitation.

Only three studies of NIV-assisted training programmes have evaluated quality of life. In patients with moderately severe COPD randomised to NIV, no additional benefit from mask NIV on exercise tolerance or health status compared with training alone was seen [29]. In another study enrolling patients with more severe lung disease, additional improvements in exercise endurance were achieved in those randomised to NIV but this did not translate into significant differences in quality of life compared with the control group [24]. In contrast, in a small, uncontrolled study, BARAKAT et al. [30] found that NIV during exercise sessions led to improved exercise tolerance and decreased breathlessness during exercise as well as improved quality of life. The current evidence suggests that NIV is feasible and beneficial in achieving better clinical and physiological outcomes from exercise training in selected patients with moderate-to-severe COPD with marked dyspnoea. However, future studies need to evaluate the impact such an approach has on quality of life and postrehabilitation physical activity levels.

\section{NIV as part of a training programme: chest wall restrictive disorders}

Interventions that allow patients with severe chest wall restriction to exercise at higher intensities for a longer duration could achieve greater improvements postexercise training, as shown in patients with COPD. While studies examining the acute effects of NIV during exercise in people with severe chest wall restriction have demonstrated an increase in exercise capacity compared with exercise without NIV, to date, no study has been published that determines whether NIV in conjunction with exercise training can confer benefits over and above exercise training alone. Therefore, the clinical features of those individuals who respond to this intervention need to be investigated.

\section{Upper-limb exercise}

Upper-limb exercise is an important component of a comprehensive exercise training programme in people with COPD [1] and may improve the performance of everyday tasks involving the arms. As many chest wall muscles that are involved in the performance of upper-limb activities also assist inspiration, inspiratory effort is significantly increased by the simple act of arm elevation [31]. Therefore, strategies that reduce inspiratory effort during arm activity may allow patients to exercise for longer. However, in comparison to studies investigating the value of NIV in lower limb exercise, there are few studies looking at upper-limb exercise. PocG et al. [31] demonstrated that arm elevation significantly increased inspiratory muscle work in COPD patients with chronic respiratory failure and that NIV during arm elevation reduced dynamic intrinsic positive end expiratory pressure (PEEPi) and inspiratory muscle work while increasing minute ventilation. In a heterogeneous group of patients with chronic hypercapnic respiratory failure enrolled in an acute randomised crossover trial, NIV during unsupported arm exercise increased endurance time, reduced dyspnoea and perceived exertion and improved oxygenation compared with exercise without NIV [32]. In this study, supplemental oxygen was added during exercise testing to maintain arterial oxygen saturation measured by pulse oximetry $\left(\mathrm{Sp}_{1} \mathrm{O}_{2}\right)$ at $\sim 93 \%$ at rest. However, currently there are no published data evaluating the impact of NIV during upper-limb training in patients with chronic respiratory disease. Further research is warranted to determine whether the use of NIV during upper-limb training delivers additional clinical benefits in patients with chronic respiratory disease in terms of improved ability to perform functional tasks.

\section{NIV during functional activities}

While NIV can acutely improve exercise performance and provide additional benefits during a lower-limb exercise training programme in selected patients, there is limited data on the feasibility of using NIV during functional activities such as ground walking [32-34]. In a randomized trial DreHER et al. [33] found that plus NIV and oxygen significantly improved arterial oxygen tension $\left(\mathrm{Pa}_{1} \mathrm{O}_{2}\right)$, increased distance walked and reduced dyspnoea compared with walking with oxygen alone in patients with severe stable COPD already established on home NIV. The authors speculated that the substantial improvement in oxygenation with NIV-aided walking could reduce cardiovascular complications that may arise during exercise due to severe desaturation even when supplemental oxygen is provided. Likewise, in a randomised trial in a heterogeneous population of patients with severe stable respiratory failure, Menadue 
et al. [32] similarly found a small but significant improvement in $\mathrm{Sp}_{1} \mathrm{O}_{2}$ at rest and at isotime exercise with NIV plus supplemental oxygen compared with supplemental oxygen alone during ground walking. However, despite the improvement in gas exchange, exercise capacity was not improved. One explanation for the discrepancy in results between these two studies may be the much higher levels of pressure support used by DREHER et al. [33]: $25 \mathrm{cmH}_{2} \mathrm{O}$ compared with 10 $\mathrm{CmH}_{2} \mathrm{O}$ used by Menadue et al. [32]. This suggests that the level of pressure support provided in the latter study may have been insufficient to adequately unload inspiratory muscles and therefore improve exercise capacity [32]. Although inspiratory pressure support levels of $\sim 10 \mathrm{cmH}_{2} \mathrm{O}$ have been shown to be effective in reducing dyspnoea and improving exercise tolerance in COPD patients during treadmill running and cycling, ground walking requires a greater amount of work from larger muscle groups [32]. Consequently, greater pressure support may be required. In addition, the study of Menadue et al. [32] included patients with severe chest wall restriction from kyphoscoliosis. The available data suggest that patients with chest wall restriction require higher inflation pressures to benefit from NIV-assisted exercise [21-23, 32] compared with patients with COPD. Further work is needed to determine the effects of high and low levels of pressure support during ground walking in patients with both severe obstructive and restrictive thoracic disorders to determine the most effective levels of ventilatory assistance required to improve dyspnoea and functional activity.

The manner in which ventilatory support can be provided in order to facilitate and increase unrestricted walking also requires further investigation. In published studies to date, the ventilator has generally been attached to a rollator frame. While this may be an option indoors, it can be limiting for patients wanting more outdoor independence. Consequently, the feasibility of carrying ventilatory-assist equipment in a backpack to allow less restricted activity has been evaluated. Using a specifically designed, lightweight and portable inspiratory support device during a field endurance walk test in patients with severe COPD, a large decrease in endurance time and a tendency for higher breathlessness scores compared with unassisted walking was found [35]. More recently, DreHER et al. [34] found that the addition of NIV to supplemental oxygen during a 12-min walk test in patients with severe COPD produced better oxygenation than walking with supplemental oxygen alone, even at double the normal dosage. Despite this, no difference in dyspnoea occurred, and as with the previous study [34], walking distance was reduced. The weight of the backpack ( $7.3 \mathrm{~kg}$ ) was seen by DreHer et al. [34] as the major barrier to achieving benefits from ambulatory NIV, while inadequacy of the flow output to meet ventilatory demand during higherintensity activity was the most likely explanation for the detrimental effects of NIV during field walking in the ReviL et al. [35] study. Consequently, lightweight devices that are suitable for unrestricted ambulation need to be developed and evaluated carefully if NIV-aided daily living activities are to become a reality.

\section{How much does NIV during exercise improve exercise performance?}

Although the mechanisms underlying exercise limitation are different for individuals with severe COPD than for those with severe chest wall restriction, both groups complain of severe exertional dyspnoea and often have a ventilatory limitation to exercise. A number of features common to both disorders can contribute to dyspnoea during exercise in these two populations, including inspiratory muscle weakness, mechanical restriction of the chest wall, abnormal gas exchange, excessive loading of the inspiratory muscles and cardiovascular factors. In addition, peripheral muscle function may also be impaired. The benefits of using NIV during exercise may arise from addressing any of one or more of these factors.

Ventilatory demand is increased during exercise, increasing the workload on the inspiratory muscles to a point where fatigue may occur. Individuals with baseline respiratory muscle weakness would be more vulnerable to fatigue and therefore dyspnoea during exercise. By reducing respiratory muscle effort during exercise $[15,31]$, fatigue and dyspnoea could be decreased $[2,16]$, permitting a higher intensity of exercise for a longer period of time. The only factor significantly contributing to the improvement in exercise endurance time as a result of applying NIV in patients with COPD was baseline inspiratory muscle strength [3], although this only explained $24 \%$ of the variance in outcome seen. Nevertheless, unloading of the inspiratory muscles is an important direct mechanism by which NIV improves exercise endurance and reduces exertional dyspnoea $[3,15,16,31]$. 
The use of NIV may also permit a more advantageous pattern of breathing during exercise. In both patients with COPD and those with restrictive chest wall disorders, the ability to increase tidal volume during exercise is often severely limited. Therefore, any increase in minute ventilation is achieved through an increase in respiratory rate. In patients with COPD, the higher tidal volume and lower respiratory rate associated with the use of NIV $[16,24,26]$ would permit more effective alveolar ventilation and gas exchange [16, 26], and may limit the development of dynamic hyperinflation [16, 24], which has previously been shown to adversely impact exercise endurance [36]. In patients with severe restrictive chest wall disorders, maximum exercise ventilation is reduced in proportion to a fall in vital capacity. A breathing pattern of low tidal volume with high respiratory rate during exercise promotes increased dead space ventilation, and therefore more extreme oxygen desaturation. By increasing minute ventilation through the generation of larger tidal volumes, NIV would permit better alveolar ventilation during exercise, reducing the fall in oxygen saturation and limiting symptoms, thereby improving exercise tolerance. Supporting this, several studies have found significant correlations between increased exercise duration and improvements in $\mathrm{Sp}_{1} \mathrm{O}_{2}$ and dyspnoea relief using NIV during exercise $[4,22]$.

Arterial hypoxaemia can also have a detrimental effect on exercise performance due to a reduction of oxygen supply to exercising muscles, and impairment of cardiac function. In patients with chest wall restriction, those responding to NIV had a lower oxygen pulse at the end of maximum exercise, suggesting a limited ability to increase stroke volume and hence cardiac out put during exercise [4]. By ameliorating the fall in oxygen saturation during exercise, NIV may improve exercise tolerance by reducing heart rate for a given level of cardiac output, or by reducing the contribution of hypoxic vasoconstriction to the development of exercise induced pulmonary hypertension [4].

In addition to improving oxygen delivery, NIV may also improve exercise tolerance by increasing blood supply to the peripheral exercising muscles. It has been previously shown in healthy, competitive cyclists that there is competition for blood flow between the legs and the respiratory muscles during sustained high-intensity exercise with high levels of ventilation [37]. Increasing work of breathing during exercise causes a substantial portion of cardiac output to be directed to the respiratory muscles, with a corresponding reduction in leg blood flow due to vasoconstriction [37]. This reduction in locomotor muscle perfusion coincides with increased leg muscle fatigue and has a detrimental effect on exercise performance [38]. In patients with moderate-tosevere COPD, unloading of the respiratory muscles with NIV has recently been shown to increase locomotor muscle oxygenation and improve perfusion compared with exercise with sham NIV [19]. This enhanced leg muscle oxygenation during NIV was not secondary to improved intrapulmonary gas exchange or to enhanced central haemodynamic adjustments to exercise. Rather it appeared that the respiratory muscle unloading achieved with NIV reduced the blood flow requirements of the respiratory muscles and permitted a redistribution of the available cardiac output to the peripheral working muscles [19]. Hence, using NIV to reduce work of breathing and dyspnoea during exercise may lead to improved oxygenation and perfusion of the exercising muscles, enabling more intense and prolonged exercise training sessions.

\section{Practical aspects of using NIV during exercise}

While there is a growing body of evidence to support the use of NIV as an adjunct to exercise training in patients with severe COPD and severe chest wall restriction, substantial work still needs to be undertaken to identify those individuals most likely to benefit from this intervention and to determine how best to incorporate NIV-assisted exercise training in routine clinical practice. At present, the use of NIV as a training adjunct needs to be carried out by health professionals who are familiar with the technique set up, and used in appropriate patients in whom the standard approach to pulmonary rehabilitation is not tolerated.

\section{Patient selection}

A wide response to NIV-assisted exercise has been found even among people with severe disease or a significantly reduced ventilatory capacity [2-4] highlighting the need to select patients carefully before embarking on this therapy. In a study that investigated the acute effects of NIV-assisted exercise, endurance time increased in $60 \%$ of patients but decreased in $31 \%$ of patients with severe COPD compared with unassisted exercise [3]. Similarly, in patients with chest 


\section{Table 2 Practical considerations in implementing NIV-assisted training}

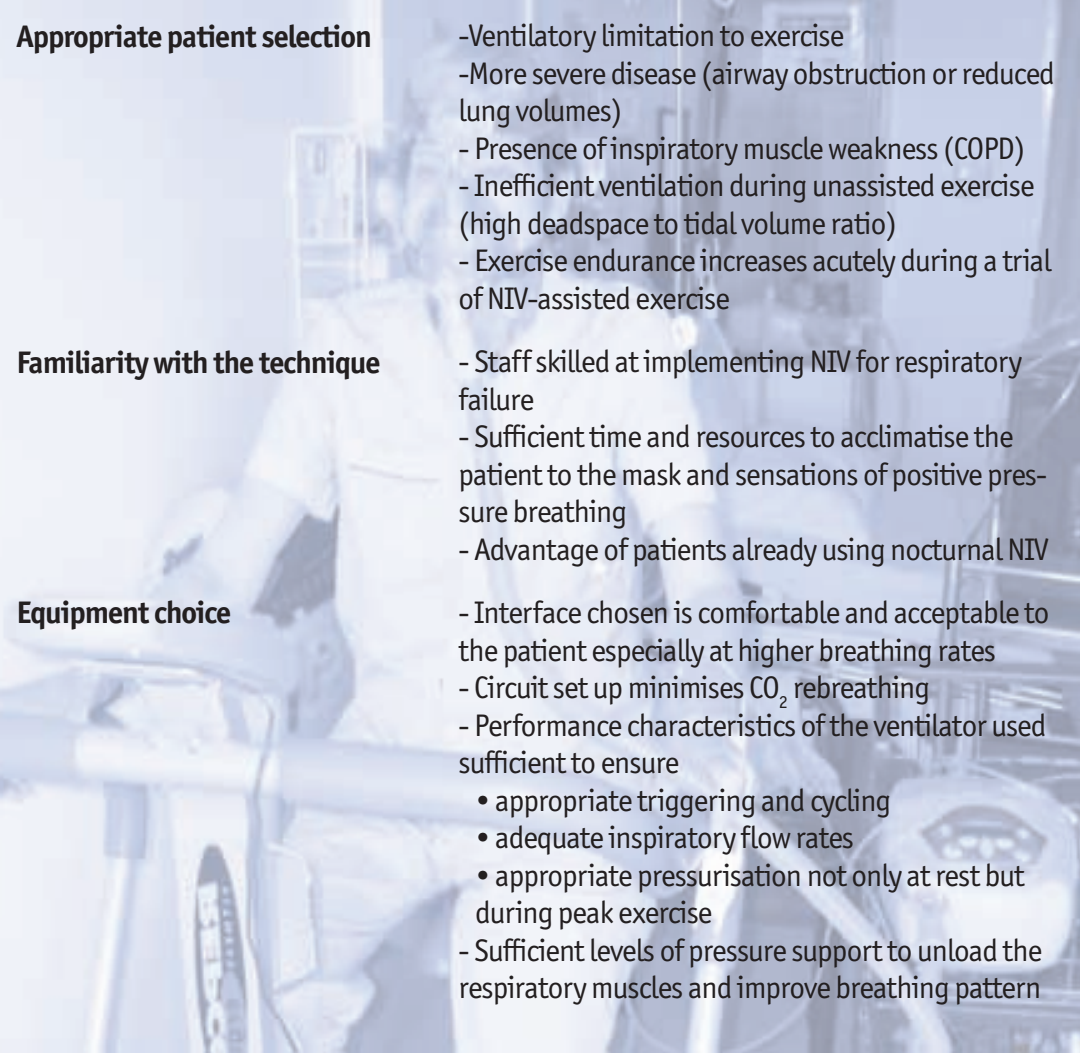

wall restriction and CRF, only half were deemed "NIV responders" [4]. Generally, it appears that patients with more severe inspiratory muscle weakness [24], and more severe obstructive [14, 29] or restrictive lung mechanics [4] are more likely to respond to therapy. Practically, patients with significant improvements in exercise performance during an acute application of NIV also tend to benefit most from training with therapy, although the predictive validity of this acute response is low (table 2) [24].

\section{Familiarity with the technique}

Despite the overall positive outcomes of NIVassisted exercise that have been reported, not all patients with severe COPD or severe chest wall restriction tolerate therapy $[18,29,30]$. Familiarity with the technique is likely to be an important factor in how well NIV-assisted exercise is tolerated. One study found no immediate benefit in using NIV in a group of 39 patients with COPD [13]. However, these patients only had a 5-10 min session to acclimatise to the mask and equipment. In contrast, other studies showing positive outcomes with NIV have undertaken practice sessions with the equipment for up to a week $[2,3$,
14, 26]. Noncompliance with NIV will be an issue if the patient finds the equipment uncomfortable or cumbersome $[26,29,34]$. Therefore, ensuring the patient is well acclimatised to therapy and using an interface which they feel comfortable with are important aspects of successfully using NIV. For patients with CRF already using nocturnal NIV, applying the technique during exercise or ambulation is often not a difficult transition [33]. Medical professionals involved in setting up NIV as an adjunct to exercise training also need to be familiar with the technique. Some investigators have reported NIV-assisted training as time consuming [29], related to supervision of individuals and the need to adjust the level of assistance if required. Therefore, use of NIV during exercise is primarily confined to centres with some degree of experience with the technique.

\section{Interfaces}

A variety of interfaces have been used during NIVassisted exercise including nasal masks $[13,14$, 29], oronasal masks $[25,26,28]$ and mouthpiece ventilation (figure 1) [3, 24]. At present no data are available to suggest one type of interface is superior to another; however, there are potential problems that might arise related to the interface that could impact on tolerance to therapy. Since mouth breathing is common during exercise [39], the use of a nasal mask may impose some difficulties for patients preferring to breathe through their mouth, resulting in leak, discomfort and ineffective triggering of the ventilator [21]. Another potential problem is carbon dioxide rebreathing, especially when a mouthpiece is used. Current face masks are designed to minimise rebreathing, with some masks able to reduce dynamic dead space to below that of physiological dead space [40]. In contrast, the use of a circuit exhalation port can create less efficient washout of carbon dioxide [41], especially at high respiratory frequencies and in the absence of endexpiratory pressure [42]. Any increase in apparatus dead space would promote higher carbon dioxide, which in turn could stimulate ventilatory drive and produce greater levels of dyspnoea without improving exercise tolerance [14].

\section{Modalities and settings}

Various modalities of NIV have been used successfully to improve exercise tolerance in patients with COPD or chest wall restriction, including CPAP, IPS, bilevel ventilatory support and PAV. PAV was designed to provide a variable level of ventilatory assistance in proportion to respiratory demand, 

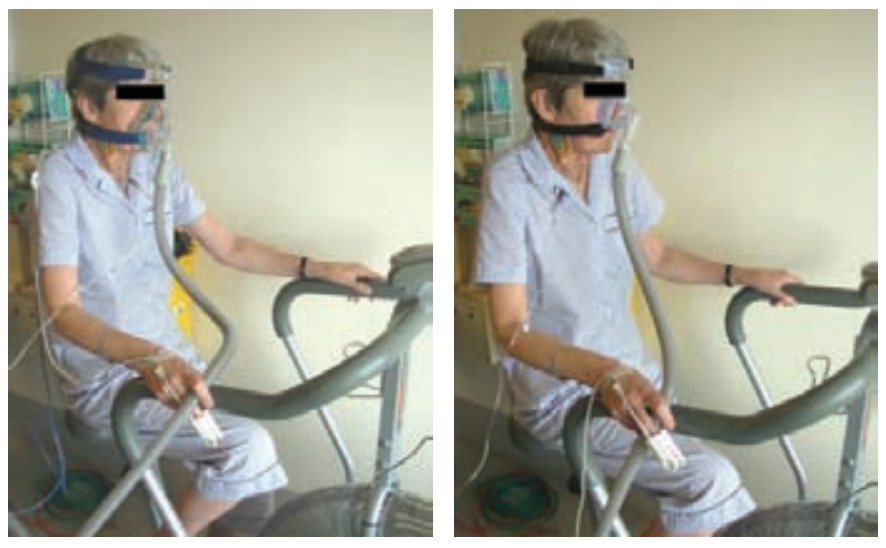

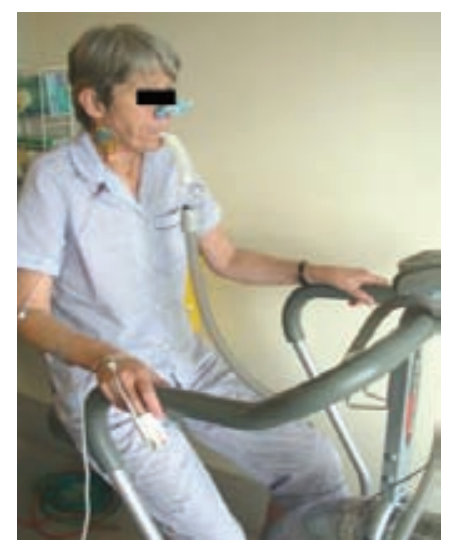

tion have used pressures of $15 \mathrm{cmH}_{2} \mathrm{O}$ or more $[4,22,23]$, whereas pressure support $\sim 10 \mathrm{cmH}_{2} \mathrm{O}$ significantly reduced distance walked compared with unencumbered exercise [21]. In a preliminary report, MenAdUe et al. [43] compared the effects of high $\left(20 \mathrm{cmH}_{2} \mathrm{O}\right)$ and low $\left(10 \mathrm{cmH}_{2} \mathrm{O}\right)$ level pressure support in a group of patients with kyphoscoliosis during treadmill walking. Endurance time was found to be significantly greater during $20 \mathrm{cmH}_{2} \mathrm{O}$ of pressure support compared with unassisted exercise, sham pressure support (CPAP $4 \mathrm{cmH}_{2} \mathrm{O}$ ) or pressure support of $10 \mathrm{cmH}_{2} \mathrm{O}$, suggesting the need for higher inflation pressures in this population to adequately unload the respiratory muscles and improve alveolar ventilation.

\section{Equipment considerations}

The performance characteristics of the ventilator used can also have a significant impact the response to exercise-assisted NIV. During exercise relatively high levels of ventilation need to be supported and this places a significant demand on the ventilator. If the ventilator is unable to meet these demands adequately, exercise performance may be impeded rather than assisted $[17,21,28,35]$. Problems may arise from delayed or ineffective triggering [21], inadequate inspiratory flow rates to deliver the preset level of pressure support [35], or dyssynchrony between the patient and the ventilator [18]. The performance characteristics of the ventilator needs to be appropriate, particularly when high levels of pressure support are being provided and respiratory rate is rapid. If the capacity of a ventilator is inadequate, the added resistance of the ventilator circuit can increase the load placed on the respiratory system and impair rather than augment exercise performance [17, 18]. Although the suitability of widely available "home" ventilators to meet the high ventilatory needs of exercise has been questioned [44], the increasing 
sophistication and capacity of many of these devices and the accumulating evidence of benefit suggest they are suitable. However, clinicians need to be aware of the performance characteristics of the devices they are using during exercise training, and establish that the device and settings are capable of meeting the patient's physiological needs throughout exercise.

When NIV is used for independent walking, the ventilator needs to be powered either from an internal battery or attached to an external power source (battery). Supplemental oxygen, if required, can be provided through a lightweight oxygen tank. At present, the most convenient way to allow patient independence is to attach the necessary equipment to a rollator [32]. Both during exercise training and independent walking, oxygen flow is adjusted to ensure $\mathrm{Sp}, \mathrm{O}_{2}$ remains above $90-92 \%$.

\section{Conclusion}

At present, there is no scientific or clinical justification to support the routine use of NIV as an adjunction to exercise training in patients with COPD or chest wall restriction. However, in carefully selected patients with a low ventilatory capacity, this approach is feasible and can provide additional benefits over exercise training alone. More work is needed to refine who will benefit from this intervention and what type of ventilatory support and settings are most appropriate. Finally, it remains to be established whether the improved exercise capacity achieved during NIV-assisted training can produce greater levels of physical activity outside a rehabilitation programme and to what extent this improves quality of life in patients with severe respiratory limitation.

\section{Educational questions}

Are the following statements true or false?

1. Unloading of the respiratory muscles appears to be an important mechanism by which NIV during exercise reduces breathlessness and improves exercise endurance.

2. Patients with less severe disease and better lung mechanics appear to respond best to NIVassisted exercise.

3. Benefits from NIV-assisted exercise have been shown during both lower limb training such as treadmill walking and cycling as well as during upper-limb exercise.

4. A variety of interfaces including mouthpiece, nasal masks and orofacial masks are suitable for NIV during exercise.

5. Inspiratory pressure support is more effective in achieving additional benefits during exercise training than other forms of NIV such as bilevel support or PAV.

6. Patients with chest wall restriction appear to require higher levels of pressure support to achieve benefits from NIV during exercise compared to patients with COPD.

7. In patients with COPD, use of NIV during exercise training has been shown to lead to substantial improvements in physical activity levels outside of the rehabilitation environment.

8. NIV-assisted training is now seen as an important part of routine management in patients with respiratory disorders participating in pulmonary rehabilitation programmes.

\section{References}

1. Ries AL, Bauldoff GS, Carlin BW, et al. Pulmonary rehabilitation: joint ACCP/AACVPR evidence-based clinical practice guidelines. Chest 2007;131: Suppl. 5, 4S-42S.

2. Keilty SEJ, Ponte J, Fleming TA, Moxham J. Effect of inspiratory pressure support on exercise tolerance and breathlessness in patients with severe stable chronic obstructive pulmonary disease. Thorax 1994; 49: 990-994.

3. van't Hul A, Gosselink R, Hollander P, Postmus P, Kwakkel G. Acute effects of inspiratory pressure support during exercise in patients with COPD. Eur Respir J 2004; 23: 34-40.

4. Borel J-C, Wuyam B, Chouri-Pontarollo N, Deschaux C, Levy P, Pépin JL. During exercise non-invasive ventilation in chronic restrictive respiratory failure. Respir Med 2008; 102: 711-719.

5. Young AC, Wilson JW, Kotsimbos TC, Naughton MT. Randomised placebo controlled trial of non-invasive ventilation for hypercapnic cystic fibrosis. Thorax 2008; 63: 72-77.

6. Schonhofer B, Ardes P, Geibel M, Köhler D, Jones PW. Evaluation of a movement detector to measure daily activity in patients with chronic lung disease. Eur Respir J 1997; 10: 2814-2819.

7. Schonhofer B, Wallstein S, Wiese C, Kohler D. Noninvasive mechanical ventilation improves endurance performance in patients with chronic respiratory failure due to thoracic restriction. Chest 2001; 119: 1371-1378.

8. Meecham Jones DJ, Paul EA, Jones PW, Wedzicha JA. Nasal pressure support ventilation plus oxygen compared with oxygen therapy alone in hypercapnic COPD. Am J Respir Crit Care Med 1995; 152: 538-544.

9. Clini, E, Sturani C, Rossi A, et al. The Italian multicentre study on noninvasive ventilation in chronic obstructive pulmonary disease patients. Eur Respir J 2002; 20: 529-538. 
22. Tsuboi T, Ohi K, Hirata H, Otsuka N, Kita H, Kuno K. Ventilatory support during exercise in patients with pulmonary tuberculosis sequelae. Chest 1997; 112: 1000-1007.

23. Vila B, Servera E, Marín J, et al. Noninvasive ventilatory assistance during exercise for patients with kyphoscoliosis. A pilot study. Am J Phys Med Rehabil 2007; 86: 672-677.

24. van't Hul A, Gosselink R, Hollander P, Postmus P, Kwakkel G. Training with inspiratory support pressure in patients with severe COPD. Eur Respir J 2006; 27: 65-72.

25. Hawkins $\mathrm{P}$, Johnson $\mathrm{LC}$, Nikoletou $\mathrm{D}$, et al. Proportional assist ventilation as an aid to exercise training in severe chronic obstructive pulmonary disease. Thorax 2002; 57: 853-859.

26. Reuveny R, Ben-Dov I, Gaides M, Reichert N. Ventilatory support during training improves training benefit in severe chronic airway obstruction. IMAJ 2005; 7: 151-155.

27. Toledo A, Borghi-Silva A, Sampaio LM, Ribeiro KP, Baldissera V, Costa D. The impact of noninvasive ventilation during the physical training in patients with moderate-to-severe chronic obstructive pulmonary disease (COPD). Clinics 2007; 62: 113-120.

28. Costes F, Agresti A, Court-Fortune I, Roche F, Vergnon JM, Barthélémy JC. Noninvasive ventilation during exercise tolerance in patients with chronic obstructive pulmonary disease. J Cardiopulm Rehabil 2003; 23: 307-313.

29. Bianchi L, Foglio K, Porta R, Baiardi R, Vitacca M, Ambrosino N. Lack of additional effect of adjunct of assisted ventilation to pulmonary rehabilitation in mild COPD patients. Respir Med 2002; 96: 359-367.

30. Barakat S, Michele G, Nesme P, Nicole V, Guy A. Effect of a noninvasive ventilatory support during exercise of a program in pulmonary rehabilitation in patients with COPD. Intern J COPD 2007; 2: 585-591.

31. Poggi R, Appendini L, Polese G, Colombo R, Donner CF, Rossi A. Noninvasive proportional assist ventilation and pressure support ventilation during arm elevation in patients with chronic respiratory failure. A preliminary, physiologic study. Respir Med 2006; 100: 972-979.

32. Menadue C, Alison JA, Piper AJ, Flunt D, Ellis FR. Non-invasive ventilation during arm exercise and ground walking in patients with chronic hypercapnic respiratory failure. Respirology 2009; 14: 251-259.

33. Dreher M, Storre JH, Windisch W. Noninvasive ventilation during walking in patients with severe COPD: a randomised cross-over trial. Eur Respir J 2007; 29: 930-936.

34. Dreher M, Doncheva E, Schwoerer A, et al. Preserving oxygenation during walking in severe chronic obstructive pulmonary disease: noninvasive ventilation versus oxygen therapy. Respiration 2008 [Epub ahead of print. PMID: 19092234].

35. Revill, SM, Singh SJ, Morgan MD. Randomized controlled trial of ambulatory oxygen and an ambulatory ventilator on endurance exercise in COPD. Respir Med 2000; 94: 778-783.

36. Porszasz J, Emtner M, Goto S, Somfay A, Whipp BJ, Casaburi R. Exercise training decreases ventilatory requirements and exercise-induced hyperinflation at submaximal intensities in patients with COPD. Chest 2005; 128: 2025-2034

37. Harms CA, Babcock MA, McClaran SR, et al. Respiratory muscle work compromises leg blood flow during maximal exercise. J Appl Physiol 1997; 82: 1573-1583.

38. Harms CA, Wetter TJ, St Croix CM, Pegelow DF, Dempsey JA. Effects of respiratory muscle work on exercise performance. J Appl Physiol 2000; 89: 131-138.

39. Wheatley JR, Amis TC, Engel LA. Oronasal portioning of ventilation during exercise in humans. J Appl Physiol 1991; 71: 546-551.

40. Saatci E, Miller DM, Stell IM, Lee KC, Moxham J. Dynamic dead space in face masks used with noninvasive ventilators: a lung model study. Eur Respir J 2004; 23: 129-135.

41. Schettino GPP, Chatmongkolchart, Hess DR, Kacmarek RM. Position of exhalation port and mask design affect $\mathrm{CO}_{2}$ rebreathing during noninvasive positive pressure ventilation. Crit Care Med 2003; 31: 2178-2182.

42. Lofaso F, Brochard L, Touchard D, Hang A, Isabey D. Evaluation of carbon dioxide rebreathing during pressure support ventilation with airway management system (BiPAP) devices. Chest 1995; 108: 772-778.

43. Menadue C, Alison J, Piper A, Hollier C, Flunt D, Ellis E. Effect of high and low level pressure support during walking in patients with severe kyphoscoliosis. Eur Respir J 2008; 32: Suppl 52, S333.

44. Ambrosino N. Assisted ventilation as an aid to exercise training: a mechanical doping? Eur Respir J 2006; 27: 3-5. 